\title{
Reinhold Reith, Umweltgeschichte in der Frühen
}

Neuzeit

München : Oldenbourg (Enzyklopädie der Geschichte, 85), 2011, 196 p., $29,90 €$

Guillaume Garner

\section{Q OpenEdition}

Journals

Édition électronique

URL : http://journals.openedition.org/ifha/7713

DOI : 10.4000/ifha. 7713

ISSN : 2198-8943

Éditeur

IFRA - Institut franco-allemand (sciences historiques et sociales)

Référence électronique

Guillaume Garner, «Reinhold Reith, Umweltgeschichte in der Frühen Neuzeit », Revue de I'IFHA [En ligne], Date de recension, mis en ligne le 13 décembre 2013, consulté le 22 septembre 2020. URL : http:// journals.openedition.org/ifha/7713; DOI : https://doi.org/10.4000/ifha.7713

Ce document a été généré automatiquement le 22 septembre 2020

(CIFHA 


\title{
Reinhold Reith, Umweltgeschichte in der Frühen Neuzeit
}

\author{
München : Oldenbourg (Enzyklopädie der Geschichte, 85), 2011, 196 p., \\ $29,90 €$
}

Guillaume Garner

En Allemagne comme dans les pays anglo-saxons, les questions environnementales sont depuis les années 1980 devenues un thème majeur de la recherche historique. Cet essor des travaux, dans des directions multiformes et selon des approches extrêmement variables, suscite la parution de synthèses, destinées entre autres à un public étudiant, faisant le point des connaissances et débats historiographiques. C'est l'objectif que s'est fixé R. Reith, suivant la tripartition habituelle de la collection «Enzyklopädie der Geschichte »: un aperçu de l'état des connaissances, une présentation de l'état de la recherche, une bibliographie thématique (d'environ 500 titres). Si la modernité s'est progressivement imposée comme période relativement pertinente pour l'histoire de l'environnement, la délimitation géographique de la matière traitée (il s'agit d'histoire allemande) comporte une part d'arbitraire dont l'auteur est conscient. Il résout ce problème en étudiant une Mitteleuropa au sens large (comprenant le Saint-Empire et la Suisse) et en multipliant les coups de projecteur sur les aires voisines (Angleterre, France, Pays-Bas, Scandinavie en particulier).

R. Reith centre son propos sur l'étude des interactions entre l'homme et la nature et divise son étude encyclopédique en distinguant un «environnement naturel » (dans lequel l'influence des éléments et phénomènes naturels est essentielle) et un « environnement anthropogène » soumis à l'influence déterminante des hommes - la partie consacrée à l'environnement naturel traitant essentiellement du climat, mais également des catastrophes naturelles (séismes, raz-de-marée) et des épidémies. Conçue à des fins pédagogiques, cette bipartition comporte évidemment une part d'arbitraire (la faune, la flore et les forêts relèvent-elles uniquement de l'environnement anthropogène ?), mais elle a le mérite d'élargir la focale (vers les relations hommes-animaux, vers les activités économiques, mais aussi vers la ville) et d'éviter de donner une place démesurée à des approches purement culturalistes. 
La troisième partie, historiographique, abandonne cette bipartition et reprend les thèmes majeurs précédemment abordés : climat, catastrophes naturelles, épidémies, forêt, sources d'énergie, ville, durabilité. Cette troisième partie se distingue par l'ampleur des thèmes abordés, notamment dans le paragraphe sur la ville comme élément de l'environnement (qui aborde par exemple la question du recyclage et de la friperie), et par une approche qui fait la part belle aux questionnaires de l'histoire économique et de l'histoire des techniques. À plusieurs reprises, R. Reith souligne que les relations entre l'homme et la nature ont à l'époque moderne une forte dimension économique et que des réponses politiques sont apportées aux défis posés, par exemple dans la gestion des ressources forestières. Les travaux relevant d'une histoire culturelle de l'environnement ne sont évidemment pas négligés, notamment à propos de la perception des catastrophes naturelles, de même que l'histoire des concepts est également prise en compte, à travers une mise au point sur la notion de durabilité (Nachhaltigkeit) et d'oeconomia naturae.

Cet ouvrage remplit ainsi parfaitement la mission qui incombe aux volumes de la collection et se distingue avant tout par l'ampleur et la diversité des thèmes abordés, le tout dans une langue qui sait rester pédagogique.

INDEX

Index chronologique : Frühe Neuzeit

Thèmes : Umweltgeschichte

\section{AUTEUR}

GUILLAUME GARNER

ENS Lyon/UMR 5190 LARHRA 